\title{
FUNCTIONS OF BOUNDED EXPANSION: NORMAL AND BLOCH FUNCTIONS
}

\author{
P. M. GAUTHIER and J. XIAO
}

(Received 3 April 1998; revised 2 October 1998)

\author{
Communicated by P. C. Fenton
}

\begin{abstract}
Normal functions and Bloch functions are respectively functions of bounded spherical expansion and bounded Euclidean expansion. In this paper we discuss the behaviour of normal functions and of Bloch functions in terms of the maximal ideal space of $H^{\infty}$, the Bergman projection and the Ahlfors-Shimizu characteristic.
\end{abstract}

1991 Mathematics subject classification (Amer. Math. Soc.): primary 30D35, 30D45, 30 D55.

\section{Introduction}

A meromorphic function $f$ on the open unit disc $D=\{z \in \mathbf{C}:|z|<1\}$ of the complex plane $\mathbf{C}$ is called normal if

$$
\|f\|_{N}=\sup _{z \in D}\left(1-|z|^{2}\right) f^{\sharp}(z)<\infty,
$$

where $f^{\sharp}(z)$ is the spherical derivative of $f$ at $z$, which is invariant under spherical rotations of $f$. Normal functions were first studied by Noshiro [16]. They were rediscovered by Lehto and Virtanen [15] who named them and discovered some of their deep properties. We shall denote by $N$ the class of all meromorphic normal functions in $D$. It was shown by Lappan [13] that $N$ is not a linear space. However, $N$ contains an important linear subset, the class $B$ of holomorphic Bloch functions, which consists of all holomorphic functions in $D$ for which

$$
\|f\|_{B}=\sup _{z \in D}\left(1-|z|^{2}\right)\left|f^{\prime}(z)\right|<\infty .
$$

Research supported in part by FCAR (Québec), NNSFC 19771006 (China), and NSERC (Canada).

(C) 1999 Australian Mathematical Society 0263-6115/99 \$A2.00+0.00 
In fact, $B$ endowed with the norm $\|f\|=\|f\|_{B}+|f(0)|$ is a Banach space. In spite of this essential difference, $N$ and $B$ behave similarly in many respects.

Let $Z$ and $W$ be smooth manifolds with metrics $\rho$ and $\sigma$ respectively. Let $f: Z \rightarrow$ $W$ be a smooth mapping. For each $z \in Z$, we have the induced linear transformation (df $)_{z}$ from the normed tangent space $\left(T_{z}, \rho_{z}\right)$ at $z$ to the normed tangent space $\left(T_{w}, \sigma_{w}\right)$ at $w=f(z)$. We define the expansion factor of $f$ at $z$ to be the norm $\left\|(d f)_{z}\right\|$ of the tangent mapping at $z$ and we shall say that the mapping $f$ is of bounded expansion if $\sup \left\{\left\|(d f)_{z}\right\|: z \in Z\right\}<\infty$. Now let $Z$ be the unit disc $D$ endowed with the hyperbolic metric and let $W$ be the Riemann sphere $\overline{\mathbf{C}}$ endowed with the spherical metric (respectively, the finite complex plane $\mathbf{C}$ endowed with the Euclidean metric). In this case we speak of functions of bounded spherical (respectively, Euclidean) expansion. From $\|\cdot\|_{N}$ (respectively $\|\cdot\|_{B}$ ), we see that a meromorphic (respectively holomorphic) function in $D$ is normal (respectively Bloch) if and only if it is of bounded spherical (respectively Euclidean) expansion. Hence, the classes $N$ and $B$ have extremely natural geometric characterizations.

The main purpose of this paper is to explore some new analogous characterizations of the spaces $N$ and $B$ by means of the maximal ideal space of $H^{\infty}$ (the space of bounded holomorphic functions on $D$ ) and the Ahlfors-Shimizu characteristic function. On the other hand, we shall point out some differences between $N$ and $B$ in terms of the Bergman projection.

This paper is organized as follows. In Section 2, we study the boundary behaviour of $\left(1-|z|^{2}\right) f^{\sharp}(z)$ and $\left(1-|z|^{2}\right) f^{\prime}(z)$ for $f$ in $N$ and $B$ respectively. In Section 3 we consider the boundary behaviour of $f$ in $N$ and $B$ alternately. In Section 4 we show that the Bloch space $B$ is equal to the Bergman projection of the space $B M O$ (functions of bounded mean oscillation on $D$ ) but the space $N$ has no such property. In Section 5 we find that the Ahlfors-Shimizu characteristic function reveals new resemblances in the appearance or nature of the spaces $N$ and $B$.

\section{Boundary behaviour of derivatives}

As usual, $H^{\infty}$ denotes the Banach algebra of all bounded holomorphic functions on $D$ with the norm

$$
\|f\|_{H^{\infty}}=\sup _{z \in D}|f(z)|<\infty .
$$

Let $M$ denote the maximal ideal space of $H^{\infty}$, which consists of all (complex) multiplicative linear functionals on $H^{\infty}$. For basic properties of the maximal ideal space $M$, we refer to [12] and [11]. With the Gelfand topology, $M$ is a compact Hausdorff space. The disc $D$ can be viewed as a subset of $M$ by identifying each 
point of $D$ with the multiplicative linear functional given by evaluation at this point, and hence the Gelfand topology coincides with the Euclidean topology on $D$. The remarkable Corona Theorem of Carleson [9] asserts that $D$ is dense in $M$ with respect to the Gelfand topology. Thus, if a function $f$ on $D$ has a continuous extension to $M$ (or any subset of $M$ ), then this extension is unique. Denote by $\hat{f}$ the maximal spherically continuous extension of $f$. That is, $\hat{f}$ is the natural extension of $f$ to the set of all points $m \in M \backslash D$ for which the cluster set $C(f, m)$ is a singleton. Denote by $C(E)$ the family of continuous complex-valued functions on $E$, where $E$ is a subset of $M$. Of course, any $f \in C(E)$ is also spherically continuous. Conversely, if $f$ is spherically continuous on $E$ and finite-valued, then $f \in C(E)$.

Recall that $M$ is contained in the unit ball of the dual of $H^{\infty}$. Two points $m_{1}, m_{2} \in$ $M$ are said to lie in the same Gleason part [12] if and only if

$$
\sup \left\{\left|\hat{f}\left(m_{2}\right)\right|: f \in H^{\infty},\|f\|_{H^{\infty}} \leq 1, \hat{f}\left(m_{1}\right)=0\right\}<1 .
$$

The Gleason part of a point $m \in M$ is denoted by $P(m)$. Hoffman showed that $P(m)$ is either a singleton or an analytic disc. In the latter case the point $m$ is said to be regular and the part $P(m)$ nontrivial. We denote by $G$ the set of all regular points in $M$. Note that $D \subset G$.

Although $G$ is not compact, Axler and Zhu [5] proved that every continuous bounded complex-valued function on $D$ has a continuous bounded extension to $G$ and they gave a sufficient condition for an extension to be of this nature. Namely, they showed the following.

LEMMA 1. Let $f: D \rightarrow \mathbf{C}$ be continuous.

(i) If $\hat{f} \in C(G)$, then $f$ is bounded on $G$.

(ii) Conversely, if $f$ is bounded and

$$
\sup _{z \in D}\left(1-|z|^{2}\right) \varlimsup_{w \rightarrow z}\left|\frac{f(w)-f(z)}{w-z}\right|<\infty,
$$

then $\hat{f} \in C(G)$.

Recall that $\hat{f} \in C(G)$ means that $\hat{f}$ is (continuously) defined and finite on $G$. This lemma is due to Axler and Zhu (see [5, Proposition 14 and Lemma 15]). Their proof depends on Lappan's paper [14, Theorems 1 and 3] and also an idea of Brown and Gauthier [8].

THEOREM 1. Let $f$ be meromorphic on $D$ and set $u(z)=\left(1-|z|^{2}\right) f^{\sharp}(z), z \in D$. Then, $f \in N$ if and only if $u$ has a continuous extension to $G$, that is $\hat{u} \in C(G)$. 
ProOF. Sufficiency. Suppose $u$ has a continuous extension $\hat{u}$ to $G$. We have noted earlier that, since $D$ is dense in $M$, this extension is unique. That is, $\hat{u}$ is (defined and) finite on $G$ and hence $\hat{u} \in C(G)$. Then, by the previous lemma, $u$ is bounded on $D$, that is, $\left(1-|z|^{2}\right) f^{\sharp}(z)=O(1)$, that is $f \in N$.

Necessity. Assume that $f \in N$. Then, $u$ is bounded and, in order to show that $u$ has an extension $\hat{u} \in C(G)$, it is enough to show that (1) holds for $u$.

Set

$$
\begin{aligned}
& s_{0}=\sup _{|f(z)| \leq 1}\left(1-|z|^{2}\right) \varlimsup_{w \rightarrow z}\left|\frac{u(w)-u(z)}{w-z}\right|, \\
& s_{\infty}=\sup _{|f(z)| \geq 1}\left(1-|z|^{2}\right) \varlimsup_{w \rightarrow z}\left|\frac{u(w)-u(z)}{w-z}\right| .
\end{aligned}
$$

Observe that

$$
\sup _{z \in D}\left(1-|z|^{2}\right) \varlimsup_{w \rightarrow z}\left|\frac{u(w)-u(z)}{w-z}\right|=\max \left\{s_{0}, s_{\infty}\right\}
$$

Since

$$
\left(\frac{1}{f}\right)^{\sharp}(z)=f^{\sharp}(z)
$$

it suffices to estimate $s_{0}$ above. To this end, we first establish a useful inequality.

Claim : If $f \in N$ and $|f(z)| \leq 1$ at a point $z \in D$, then

$$
\frac{\left(1-|z|^{2}\right)^{2}\left|f^{\prime \prime}(z)\right|}{1+|f(z)|^{2}} \leq \frac{2}{\tanh ^{2}\left(\pi /\left(4\|f\|_{N}\right)\right)}+2\|f\|_{N}^{2}+2\|f\|_{N} .
$$

In fact, fix a point $z_{0} \in D$ and set

$$
g(z)=\frac{f(\varphi(z))-f\left(z_{0}\right)}{1+\overline{f\left(z_{0}\right)} f(\varphi(z))}, \quad \varphi(z)=\frac{z+z_{0}}{1+\bar{z}_{0} z} .
$$

Then, $g(0)=0$ and $g$ is $f$ preceded by a Möbius transformation and followed by a rotation of the sphere, and so $g \in N$ with $\|g\|_{N}=\|f\|_{N}$. For $z \in D$, let $\ell=[0, z]$ be the segment from 0 to $z$. Then,

$$
\begin{aligned}
\arctan |g(z)| & =\int_{0}^{|g(z)|} \frac{d t}{1+t^{2}} \leq \int_{g(\ell)} \frac{|d w|}{1+|w|^{2}} \\
& =\int_{\ell}\left(1-|\zeta|^{2}\right) g^{\sharp}(\zeta) \frac{|d \zeta|}{1-|\zeta|^{2}} \leq \frac{\|f\|_{N}}{2} \ln \frac{1+|z|}{1-|z|}
\end{aligned}
$$


It follows that $|g(z)|<1$ for $|z|<r=\tanh \pi /\left(4\|f\|_{N}\right)$, and hence $h(\zeta)=g(r \zeta)$ is a holomorphic function from $D$ into itself. Thus, an elementary calculation gives that

$$
\begin{aligned}
r^{2} \frac{\left(1-\left|z_{0}\right|^{2}\right)^{2}\left|f^{\prime \prime}\left(z_{0}\right)\right|}{1+\left|f\left(z_{0}\right)\right|^{2}} & \leq r^{2}\left(\left|g^{\prime \prime}(0)\right|+2\|f\|_{N}^{2}+2\|f\|_{N}\right) \\
& \leq\left|h^{\prime \prime}(0)\right|+r^{2}\left[2\|f\|_{N}^{2}+2\|f\|_{N}\right] \leq 2+r^{2}\left[2\|f\|_{N}^{2}+2\|f\|_{N}\right],
\end{aligned}
$$

which yields (2).

Now, let us return to estimating $s_{0}$. Let $z \in D$ with $|f(z)| \leq 1$. Then,

$$
\begin{aligned}
\varlimsup_{w \rightarrow z} & \left|\frac{u(w)-u(z)}{w-z}\right| \\
\leq & \varlimsup_{w \rightarrow z}\left(1-|w|^{2}\right)\left|\frac{f^{\sharp}(w)-f^{\sharp}(z)}{w-z}\right| \\
& +f^{\sharp}(z) \varlimsup_{w \rightarrow z}\left|\frac{\left(1-|w|^{2}\right)-\left(1-|z|^{2}\right)}{w-z}\right| \\
\leq & \varlimsup_{w \rightarrow z}\left(1-|w|^{2}\right) \frac{|| f^{\prime}(w)\left|/\left(1+|f(w)|^{2}\right)-\right| f^{\prime}(z)\left|/\left(1+\mid f(z)^{2}\right)\right|}{|w-z|}+2 f^{\sharp}(z) \\
\leq & \varlimsup_{w \rightarrow z} \frac{1-|w|^{2}}{1+|f(w)|^{2}} \frac{\left|f^{\prime}(w)-f^{\prime}(z)\right|}{|w-z|} \\
& +\varlimsup_{w \rightarrow z} \frac{\left(1-|w|^{2}\right)\left|f^{\prime}(z)\right|\left|1 /\left(1+|f(w)|^{2}\right)-1 /\left(1+|f(z)|^{2}\right)\right|}{|w-z|}+2 f^{\sharp}(z) \\
\leq & \frac{\left(1-|z|^{2}\right)\left|f^{\prime \prime}(z)\right|}{1+|f(z)|^{2}}+\frac{2\left(1-|z|^{2}\right)\left|f^{\prime}(z)\right|^{2}|f(z)|}{\left(1+|f(z)|^{2}\right)^{2}}+2 f^{\sharp}(z) \\
\leq & \frac{\left(1-|z|^{2}\right)\left|f^{\prime \prime}(z)\right|}{1+|f(z)|^{2}}+2 \frac{\left(1-|z|^{2}\right)\left|f^{\prime}(z)\right|^{2}}{\left(1+|f(z)|^{2}\right)^{2}}+2 f^{\sharp}(z) .
\end{aligned}
$$

Consequently, from the above claim, we have that

$$
s_{0} \leq 2\left(\frac{1}{\tanh ^{2}\left(\pi /\left(4\|f\|_{N}\right)\right)}+2\|f\|_{N}^{2}+2\|f\|_{N}\right) .
$$

Of course, when $z \in D$ and $|f(z)| \geq 1$, we may consider $1 / f(z)$ and obtain the same estimate. Therefore, (1) holds for $f \in N$. This completes the proof.

A function $f$ meromorphic in $D$ is said to be in the little normal space $N_{0}$, if

$$
\lim _{|z| \rightarrow 1}\left(1-|z|^{2}\right) f^{\sharp}(z)=0 .
$$

We could also say that $f \in N_{0}$ if and only if $f$ is of vanishing spherical expansion. Let $\partial D$ denote the unit circle $|z|=1$ and $\bar{D}=D \cup \partial D$, the closed unit disc $|z| \leq 1$. 
Let $u$ be a function defined on $D$ and let $\zeta \in \partial D$. If $\hat{u}(m)$ is defined and takes the same value for every point $m \in M \backslash D$ which lies in the fiber over $\zeta$, then we can define $\hat{u}(\zeta)$ as this common value. Let $C_{0}(\vec{D})$ denote the space of functions which are continuous on $\bar{D}$ and vanish on $\partial D$.

REMARK 1. Let $f$ be meromorphic on $D$ and set $u(z)=\left(1-|z|^{2}\right) f^{\sharp}(z)$. Then, clearly, $f \in N_{0}$ if and only if $\hat{u} \in C_{0}(\bar{D})$.

For holomorphic functions, Axler and Zhu obtained the following stronger result [5, Theorem 16].

THEOREM 2. Let $f$ be a function holomorphic on $D$, let $n \geq 1$ be an integer and set $u(z)=\left(1-|z|^{2}\right)^{n} f^{(n)}(z)$. Then $f \in B$ if and only if $u$ has a continuous extension to $G$, that is $\hat{u} \in C(G)$.

Recall that a function $f$ holomorphic on $D$ is said to be in the little Bloch space $B_{0}$, if

$$
\lim _{|z| \rightarrow 1}\left(1-|z|^{2}\right) f^{\prime}(z)=0 .
$$

We could also say that $f \in B_{0}$ if and only if $f$ is of vanishing Euclidean expansion. $[28$, Theorem 5.26] tells us the following.

REMARK 2. Let $f$ be holomorphic on $D$; let $n \geq 1$ be an integer and set $u(z)=$ $\left(1-|z|^{2}\right)^{n} f^{(n)}(z)$. Then $f \in B_{0}$ if and only if $u \in C_{0}(\bar{D})$.

QUESTION 1. Do Theorem 1 and its Remark 1 have versions for higher derivatives, similar to Theorem 2 and its Remark 2?

\section{Boundary behaviour of functions}

In this section we discuss the boundary behaviour of functions in the classes $N$ and $B$.

For $z, w \in D$, let $\lambda(z, w)$ (respectively $\rho(z, w)$ ) denote the pseudohyperbolic (respectively hyperbolic) distance between $z$ and $w$.

The following characterization of $H^{\infty}$ is essentially due to Hoffman [12].

THEOREM 3. Let $f$ be holomorphic on $D$. Then, the following statements are equivalent.

(i) $f \in H^{\infty}$. 
(ii) $f$ can be extended continuously to $M$, that is $\hat{f} \in C(M)$.

(iii) $f$ is uniformly continuous with respect to the pseudohyperbolic distance. In fact, there is a constant $C$, depending only on $f$, such that

$$
|f(z)-f(w)| \leq C \lambda(z, w) \text {. }
$$

PROOF. (iii) $\Rightarrow$ (i) is obvious. (i) $\Rightarrow$ (iii) follows from applying Schwarz's lemma to the function

$$
g_{w}(\zeta)=f\left(\frac{w-\zeta}{1-\bar{w} \zeta}\right)-f(w)
$$

(i) $\Leftrightarrow$ (ii) is a well known fact due to Hoffman.

For $N$, we have the following analogous characteterization, where the Euclidean distance $|\cdot|$ is replaced by the spherical distance $\chi$ and the pseudohyperbolic distance by the hyperbolic distance.

THEOREM 4. Let $f$ be meromorphic on $D$. Then, the following statements are equivalent.

(i) $f \in N$.

(ii) $f$ can be extended spherically continuously to $G$, that is, $\hat{f}$ is defined on $G$.

(iii) $f$ is uniformly spherically continuous with respect to the hyperbolic metric. In fact, there is a constant $C$, depending only on $f$, such that

$$
\chi(f(z), f(w)) \leq C \rho(z, w) .
$$

ProOF. (i) $\Leftrightarrow$ (ii) is due to Brown and Gauthier [8]. (i) $\Leftrightarrow$ (iii) is worked out essentially by Lappan (see the proof of [14, Theorem 3]). In fact, this follows from the fact that functions in $N$ are precisely functions of bounded spherical expansion.

Brown and Gauthier also showed that this result is sharp in the following sense. They constructed a meromorphic normal function $f_{0}$ whose cluster set at each boundary point $m \in M \backslash G$ is the entire Riemann sphere $\overline{\mathbf{C}}$. Thus, for this function $f_{0}$, the natural domain of definition of $\hat{f_{0}}$ is precisely $G$; in fact, the worst possible discontinuity is achieved at all points of $M \backslash G$. Moreover, the following proposition asserts that such normal functions are actually dense in the space of all meromorphic functions on $D$ equipped with the topology of uniform convergence on compacta. A neighbourhood basis of a meromorphic function $f$ is constructed as follows. Let $E$ be a compact subset of $D$ and let $\epsilon>0$. Then, a basic neighbourhood $V(f, E, \epsilon)$ consists of all meromorphic functions $g$ on $D$ having the same poles with the same principal parts as $f$ on $E$ and such that $|f-g|<\epsilon$ on $E$. For the sake of brevity, we shall call this topology the uniform topology. 
PROPOSITION 1. For each function $f$ meromorphic on $D$ and for each neighbourhood $V(f, E, \epsilon)$ of $f$, there is a meromorphic normal function $g$ such that

(i) $g \in V(f, E, \epsilon)$,

(ii) $C(g, m)=\overline{\mathbf{C}}$, for each $m \in M \backslash G$.

PROOF. Let $f_{0}$ be the normal function mentioned above, whose cluster set at each point of $M \backslash G$ is the entire Riemann sphere $\overline{\mathbf{C}}$. Let $z_{j}, j=1, \ldots, n$ be the poles of $f-f_{0}$ on $E$ and, for each $j=1, \ldots, n$, let

$$
p_{j}\left(\frac{1}{z-z_{j}}\right)
$$

be the principal part of $f-f_{0}$ at the pole $z_{j}$. Set

$$
p(z)=\sum_{j=1}^{n} p_{j}\left(\frac{1}{z-z_{j}}\right) .
$$

Then, $f-f_{0}-p$ is holomorphic on the compact set $E$ and so, by Runge's theorem, there is a rational function $r$, having no poles on $E$ nor on the unit circle $|z|=1$, such that $\left|\left(f-f_{0}-p\right)-r\right|<\epsilon$ on $E$. Now set $g=f_{0}+(p+r)$. Since $g$ is the sum of a normal function and a rational function with no poles on the unit circle, it follows from Theorem 4 that $g$ is normal. By construction, $g$ satisfies (i). Finally, $g$ satisfies (ii) since $f_{0}$ satisfies (ii) and since $p+r$ is continuous and finite valued at each point of the unit circle and, a fortiori, at each point $m \in M$. This completes the proof.

The previous proposition is in terms of the uniform topology. The other natural topology on the space of meromorphic functions is the topology which is associated to spherically uniform convergence on compacta. For brevity, we shall call this the spherically uniform topology. Fix a metric $\chi$ on the Riemann sphere $\overline{\mathbf{C}}$. Then, in the spherically uniform topology, a basic neighbourhood $V(f, E, \epsilon)$ consists of all meromorphic functions $g$ on $D$ such that $\chi(f, g)<\epsilon$ on $E$. It is easy to see that these two topologies are different; indeed, the sequence $(z-1 / n)^{-1}$ converges to $1 / z$ in the spherically uniform topology but not in the uniform topology. On the other hand, on compacta, uniform convergence implies spherically uniform convergence, and so the uniform topology is (strictly) stronger than the spherically uniform topology. It follows that any family of meromorphic functions which is dense in the uniform topology is automatically dense in the spherically uniform topology. Thus, the above proposition, which is stated in terms of the uniform topology, also holds in the spherically uniform topology.

LEMMA 2. Let $\left\{g_{\alpha}\right\}$ be a bounded net of holomorphic functions on D and suppose $g_{\alpha} \rightarrow g$. Then, $g$ is holomorphic and $g_{\alpha}^{\prime} \rightarrow g^{\prime}$. 
PROOF. The fact that $g$ is holomorphic is well known. However, that we may interchange the limit with derivation is perhaps less well known, so we give a proof. Fix $z_{0} \in D$ and $\epsilon>0$ and write

$$
\begin{aligned}
g^{\prime}\left(z_{0}\right) & -g_{\alpha}^{\prime}\left(z_{0}\right) \\
= & g^{\prime}\left(z_{0}\right)-\frac{g(z)-g\left(z_{0}\right)}{z-z_{0}}+\frac{g(z)-g\left(z_{0}\right)}{z-z_{0}} \\
& -\frac{g_{\alpha}(z)-g_{\alpha}\left(z_{0}\right)}{z-z_{0}}+\frac{g_{\alpha}(z)-g_{\alpha}\left(z_{0}\right)}{z-z_{0}}-g_{\alpha}^{\prime}\left(z_{0}\right) \\
= & A_{0}+B_{\alpha}+A_{\alpha} .
\end{aligned}
$$

Since $\left\{g_{\alpha}\right\}$ is uniformly bounded, it follows from the Cauchy formula that the derivatives are uniformly bounded on compacta. Thus, since

$$
\left|A_{\alpha}\right|=\left|\frac{1}{z-z_{0}} \int_{z_{0}}^{z}\left[g_{\alpha}^{\prime}(\zeta)-g_{\alpha}^{\prime}\left(z_{0}\right)\right] d \zeta\right| \leq 2 \max _{\zeta \in\left[z, z_{0}\right]}\left|g_{\alpha}^{\prime}(\zeta)\right|
$$

we may choose $z \in D$ such that $\left|A_{\alpha}\right|<\epsilon / 3$, for all $\alpha$ and also $\left|A_{0}\right|<\epsilon / 3$. For this $z$, there exists an $\alpha_{\epsilon}$ such that $\alpha \geq \alpha_{\epsilon}$ implies that $\left|B_{\alpha}\right|<\epsilon / 3$. Hence, $\alpha \geq \alpha_{\epsilon}$ implies that $\left|g^{\prime}\left(z_{0}\right)-g_{\alpha}^{\prime}\left(z_{0}\right)\right|<\epsilon / 3$. This proves the lemma.

Of course, the lemma also holds for locally bounded pointwise convergence. A similar result holds for meromorphic functions provided we allow the constant $\infty$ as a meromorphic function. Let $\left\{g_{\alpha}\right\}$ be a net of meromorphic functions on $D$ and suppose $g_{\alpha} \rightarrow g$ spherically. We shall say that the net is locally spherically bounded if, for each $z_{0} \in D$, there is a proper compact subset $K \subset \overline{\mathbf{C}}$ and a neighbourhood $V_{0}$ of $z_{0}$ such that $g_{\alpha}(z) \in K$, for each $z \in V_{0}$ and for each $g_{\alpha}$. Since the spherical derivative is invariant under rotations, it is then easy to check that the following lemma is a consequence of the previous one.

LEMMA 3. Let $\left\{g_{\alpha}\right\}$ be a locally spherically bounded net of meromorphic functions on $D$ and suppose $g_{\alpha} \rightarrow g$. Then, $g$ is meromorphic and $g_{\alpha}^{\sharp} \rightarrow g^{\sharp}$.

THEOREM 5. Let $f \in N$. Then, the following statements are equivalent.

(i) $f \in N_{0}$.

(ii) $\hat{f}$ is constant on each nontrivial Gleason part in $M \backslash D$.

(iii) For any $r \in(0, \infty)$,

$$
\lim _{|w| \rightarrow 1} \sup _{\rho(z, w)<r} \frac{\chi(f(z), f(w))}{\rho(z, w)}=0 .
$$


PROOF. (i) $\Leftrightarrow$ (iii) follows readily from the definition of $N_{0}$ and Lappan's proof for [14, Theorem 3].

For (i) $\Leftrightarrow$ (ii), we recall that each nontrivial Gleason part $P(m)$ has a holomorphic structure obtained as follows $[12$, p. 75$]$. If $z_{\alpha} \in D$ converges to $m$, then

$$
L_{\alpha}(z)=\frac{z+z_{\alpha}}{1+\bar{z}_{\alpha} z}
$$

converges pointwise to $L_{m}$, a bijection of $D$ onto $P(m)$. Brown and Gauthier [8, Theorem 5] showed that, if $f$ is normal, then, on each nontrivial Gleason part $P(m)$, the function $\hat{f}$ (which is well defined by Theorem 4 ) is identically infinite or meromorphic, in the sense that $\hat{f} \circ L_{m}$ is meromorphic.

Also, we recall that Hoffman [12, p. 75] proved that a point $m \in M \backslash D$ is a regular point if and only if it lies in the closure of an interpolating sequence $\left\{z_{n}\right\}$ in $D$.

Let us return to the proof of the theorem. Brown and Gauthier concluded that $\hat{f} \circ L_{m}$ is meromorphic from the convergence $f \circ L_{\alpha} \rightarrow \hat{f} \circ L_{m}$ and the first part of the previous lemma. From the lemma, we also have that $\left(f \circ L_{\alpha}\right)^{\sharp} \rightarrow\left(\hat{f} \circ L_{m}\right)^{\sharp}$.

Sufficiency. Suppose $\hat{f}$ is constant on each nontrivial Gleason part $P(m)$ in $M \backslash D$. Then $\left(\hat{f} \circ L_{m}\right)^{\sharp}=0$. We claim that $\left(1-|z|^{2}\right) f^{\sharp}(z)$ tends to 0 as $|z| \rightarrow 1$ and hence $f \in N_{0}$. Suppose not. Then, $\left(1-\left|z_{n}\right|^{2}\right) f^{\sharp}\left(z_{n}\right)$ is bounded away from zero, for some interpolating sequence $\left\{z_{n}\right\}$. Let $m$ be any point of $M \backslash D$ in the closure of this sequence. Then, $m \in G$ and there is a subnet $\left\{z_{\alpha}\right\}$ of $\left\{z_{n}\right\}$ which converges to $m$. Now

$$
0=\left(\hat{f} \circ L_{m}\right)^{\sharp}(0)=\lim _{\alpha}\left(f \circ L_{\alpha}\right)^{\sharp}(0)=\lim _{\alpha}\left(1-|\alpha|^{2}\right) f^{\sharp}\left(z_{\alpha}\right) \text {. }
$$

This is a contradiction which proves the sufficiency.

Necessity. Assume that $f \in N_{0}$. Let $m$ be a regular point. Then $m$ lies in the closure of an interpolating sequence $\left\{z_{n}\right\}$ in $D$. Since $f \in N_{0}$,

$$
\lim _{n \rightarrow \infty}\left(1-\left|z_{n}\right|^{2}\right) f^{\sharp}\left(z_{n}\right)=0 .
$$

Fix $\epsilon>0$. By omitting finitely many terms of this sequence, we may assume that

$$
\left(1-\left|z_{n}\right|^{2}\right)\left|f^{\sharp}\left(z_{n}\right)\right|<\epsilon, \quad n=1,2, \ldots
$$

It follows, as in the proof of sufficiency, that $\left(\hat{f} \circ L_{m}\right)^{\sharp}(m)=0$. Since every point of a nontrivial part is regular, it follows that $\hat{f}$ is constant on any nontrivial part. This concludes the proof of the theorem.

Henceforth, let $d m$ denote the usual two-dimensional Lebesgue measure.

THEOREM 6. Let $f$ be holomorphic in D. Then, the following statements are equivalent. 
(i) $f \in B$.

(ii) $f$ is the Bergman projection of some function $g \in C(G)$, that is,

$$
f(z)=\frac{1}{\pi} \int_{D} \frac{g(w)}{(1-z \bar{w})^{2}} d m(w)
$$

(iii) $f$ is uniformly continuous with respect to the hyperbolic metric on D. In fact, there is a constant, depending only on $f$, such that

$$
|f(z)-f(w)| \leq C \rho(z, w)
$$

Proof. For the equivalence (i) $\Leftrightarrow$ (iii), see [24, Theorem 2.1]. In fact, this follows from the fact that Bloch functions are precisely functions of bounded Euclidean expansion. The equivalence (i) $\Leftrightarrow$ (ii) follows from the following argument. Let $f \in B$. Since all polynomials are in $B$ (even in $B_{0}$ ), we may, without loss of generality, assume that $f$ has a zero of order at least $2 n$ at 0 . Then, by [5, Lemma 4],

$$
f(z)=\frac{1}{n ! \pi} \int_{D} \frac{\left(1-|w|^{2}\right)^{n} f^{(n)}(w)}{(1-z \bar{w})^{2} \bar{w}^{n}} d m(w)=\frac{1}{\pi} \int_{D} \frac{g(w)}{(1-z \bar{w})^{2}} d m(w),
$$

where

$$
g(z)=\frac{1}{n !} \frac{\left(1-|z|^{2}\right)^{n} f^{(n)}(z)}{\bar{z}^{n}}
$$

Also, by [5, Theorem 16], it follows that $g \in C(G)$, since $f \in B$. Moreover, since $g \in C(G)$ must be bounded by Lemma 1 (i), $f$ given by (ii) belongs to $B$ (see [5, Table 2]).

The following is well known.

REMARK 3. Let $f \in H^{\infty}$. Then, the following statements are equivalent.

(i) $f \in B_{0}$.

(ii) $\hat{f}$ is finite and constant on each Gleason part in $M \backslash D$.

(iii) For any $r \in(0, \infty)$,

$$
\lim _{|w| \rightarrow 1} \sup _{\rho(z, w)<r} \frac{|f(z)-f(w)|}{\rho(z, w)}=0 .
$$

The equivalence (i) $\Leftrightarrow$ (ii) is in $[11$, p. 442] and (i) $\Leftrightarrow$ (iii) is in [25, Theorem 2.1]. 


\section{The Bergman projection}

The theorems of the previous section together with those of the present section point out some significant differences between the spaces $N, B$ and $H^{\infty}$.

For $p \in[1, \infty)$, let $L^{p}$ and $L^{\infty}$ be the Lebesgue spaces of all measurable functions $f: D \rightarrow \mathbf{C}$ with

$$
\|f\|_{L^{p}}^{p}=\int_{D}|f|^{p} d m<\infty
$$

and

$$
\|f\|_{L^{\infty}}=\underset{z \in D}{\operatorname{ess} \sup }|f(z)|<\infty,
$$

respectively. For any bounded measurable set $E \subset \mathbf{R}^{2}$ and $f \in L^{1}(E)$,

$$
f_{E}=\frac{1}{m(E)} \int_{E} f d m
$$

denotes the average of $f$ on $E$. Let $\Omega$ be a domain in $\mathbf{R}^{2}$. Following Reimann (see [20]) we define the space $B M O(\Omega)$ (bounded mean oscillation on $\Omega$ ) as the set of all functions $f \in L_{\text {loc }}^{1}(\Omega)$ satisfying

$$
\|f\|_{B M O(\Omega)}=\sup _{\Delta \subset \Omega} \frac{1}{m(\Delta)} \int_{\Delta}\left|f-f_{\Delta}\right| d m<\infty,
$$

where the supremum is taken over all Euclidean discs $\Delta \subset \Omega$. In the present paper, $B M O=B M O(D)$ stands for $B M O$ of the unit disc $D$.

We also introduce the space $V M O$ of functions of vanishing mean oscillation. These are the functions $f$ in $B M O$ for which

$$
\lim _{\delta \rightarrow 0} \sup _{m(\Delta)<\delta} \frac{1}{m(\Delta)} \int_{\Delta}\left|f-f_{\Delta}\right| d m=0 .
$$

$V M O$ is the closure of $C(\bar{D})$ in the $B M O$ norm. This fact is due to Jones (see [7, p. 310]). Using this fact, Reimann has shown (see [19]) that any function $f$ in $B M O$ of the disc can be extended to a function $\tilde{f}$ in the well known space $B M O\left(\mathbf{R}^{2}\right)$, from which it follows that

$$
L^{\infty} \subset B M O \subset \bigcap_{1 \leq p<\infty} L^{p}
$$

where the inclusions are strict. Coifman, Rochberg and Weiss [10] gave a different definition for $B M O$ of the disc, by replacing $\Delta$ by $\Delta \cap D$, where $\Delta$ ranges over all 
Euclidean discs $\Delta$ whose centers lie in $D$. Noting that, for each bounded measurable set $E \subset \mathbf{R}^{2}$, each $f \in L^{1}(E)$ and each $a \in \mathbf{C}$,

$$
\frac{1}{m(E)} \int_{E}\left|f-f_{E}\right| d m \leq \frac{2}{m(E)} \int_{E}|f-a| d m,
$$

we obtain the equivalence of the two definitions for $B M O$ of the disc by taking $E=\Delta \cap D$ and $a=\tilde{f_{\Delta}}$, where $f$ is in $B M O$ in the sense of Reimann and $\tilde{f}$ is the above mentioned extension thereof.

Moreover, $H^{\infty}, B, B_{0}$ and the Bergman spaces $L_{a}^{p}$ are the subspaces of holomorphic functions in $L^{\infty}, B M O, V M O$ and $L^{p}$ respectively. The first and last identifications are true by definition. For the middle identification, see [10].

On $L^{1}$ we define a linear operator, usually called the Bergman projection, as follows

$$
P(f)(z)=\frac{1}{\pi} \int_{D} \frac{f(w)}{(1-z \bar{w})^{2}} d m(w), \quad f \in L^{1} .
$$

It is well known $[5, \mathrm{p} .130]$ that $P$ boundedly sends $L^{p}$ onto $L_{a}^{p}$, for $p>1$ and $L^{\infty}$ onto $B$. However, we note that there cannot exist any 'projection', such as (4) mapping some class onto $N$. This is because $N$ is not a linear space, in other words, $\|\cdot\|_{N}$ is not a semi-norm. Here, we are interested in the images under $P$ of $B M O$ and $C(G)$, noting that $C(G) \subset L^{\infty} \subset B M O$, where the inclusions are strict.

THEOREM 7. $P(C(G))=P(B M O)=B$.

Proof. $P(C(G))=B$ is in fact already shown in (i) $\Leftrightarrow$ (ii) of Theorem 6. Since $B=P\left(L^{\infty}\right)$, we need only check that $P(B M O) \subset B$. Suppose $f \in P(B M O)$. Then, there is a $g \in B M O$ such that $f=P(g)$. Thus,

$$
f^{\prime \prime}(z)=\frac{6}{\pi} \int_{D} \frac{g(w) \bar{w}^{2}}{(1-\bar{w} z)^{4}} d m(w) .
$$

Since

$$
\begin{gathered}
1=\frac{1}{\pi} \int_{D} \frac{1}{(1-\bar{w} z)^{2}} d m(w), \\
0=\frac{6}{\pi} \int_{D} \frac{\bar{w}^{2}}{(1-\bar{w} z)^{4}} d m(w)=\frac{6}{\pi} \int_{D} \frac{\bar{w}^{2}}{(1-\bar{w} z)^{4}}\left(g \circ \varphi_{z}\right)_{D} d m(w),
\end{gathered}
$$

where

$$
\varphi_{z}(w)=\frac{z-w}{1-\bar{z} w}
$$


Furthermore,

$$
\begin{aligned}
f^{\prime \prime}(z) & =\frac{6}{\pi} \int_{D}\left[g(w)-\left(g \circ \varphi_{z}\right)_{D}\right] \frac{\bar{w}^{2}}{(1-\bar{w} z)^{4}} d m(w) \\
& =\frac{6}{\pi} \int_{D}\left[\left(g \circ \varphi_{z}\right)(\zeta)-\left(g \circ \varphi_{z}\right)_{D}\right] \frac{(z-\zeta)^{2}}{\left(1-|z|^{2}\right)^{2}} \frac{(1-z \bar{\zeta})^{2}}{(1-\bar{z} \zeta)^{4}} d m(\zeta) .
\end{aligned}
$$

Thus,

$$
\begin{aligned}
\left(1-|z|^{2}\right)^{2}\left|f^{\prime \prime}(z)\right| & \leq \frac{6}{\pi} \int_{D}\left|\left(g \circ \varphi_{z}\right)(\zeta)-\left(g \circ \varphi_{z}\right)_{D}\right| d m(\zeta) \\
& \leq 6\left\|g \circ \varphi_{z}\right\|_{B M O} \leq 6\|g\|_{B M O}
\end{aligned}
$$

The last inequality comes from the fact (see [19]) that $\|\cdot\|_{B M O}$ is equivalent under automorphisms of $D$. Therefore, $f \in B$ and the proof is complete.

As a consequence of the previous theorem, we have the following.

THEOREM 8. $P(C(\bar{D}))=P(V M O)=B_{0}$.

Proof. $P(C(\bar{D}))=B_{0}$ is known (see, for example [5, p. 130]). As in the previous theorem, it remains to show that $P(V M O) \subset B_{0}$. Since $P(B M O)=B$ and $P$ maps polynomials of the form

$$
\left\{z^{m} \bar{z}^{n}: m, n \text { nonnegative integers }\right\}
$$

(which are dense in $V M O$ ), to holomorphic polynomials (which are dense in $B_{0}$ ), we have $P(V M O) \subset B_{0}$, which completes the proof.

\section{The Ahlfors-Shimizu characteristic}

In this section, we shall provide criteria for functions to be in the classes $N$ and $B$ in terms of the generalized Ahlfors-Shimizu characteristic.

For $f$ meromorphic on $D$ and $r \leq 1$, let $S(f, r)$ (respectively $s(f, r)$ ) denote the spherical area of the image $f(|z|<r)$ with multiplicity taken (respectively not taken) into account. Thus,

$$
S(f, r)=\int_{|z|<r} f^{\sharp}(z) d m(z)
$$

is the spherical area of the Riemannian image over the image $f(|z|<r)$, while $s(f, r)$ is the spherical area of the image $f(|z|<r)$ itself. 
For $p \in(0, \infty)$, the generalized Ahlfors-Shimizu characteristic function of $f$ is defined as

$$
T^{*}(f, r, p)=\frac{1}{\pi} \int_{0}^{r} \log ^{p-1}\left(\frac{1}{t}\right) S(f, t) \frac{d t}{t} .
$$

In particular, $T^{*}(f, r, 1)=T^{*}(f, r)$ is the Ahlfors-Shimizu characteristic function of $f$. This quantity is related to the Nevanlinna characteristic function $T(f, r)$ via the following inequality $[21, \mathrm{p} .26]$ :

$$
\left|T^{*}(r, f)-T(r, f)-\log ^{+}\right| f(0)|| \leq \frac{1}{2} \log 2 .
$$

Stroethoff [22] gave a Nevanlinna type characterization for $B$, extending the work of Baernstein [6, Corollary 2]. We shall give an Ahlfors-Shimizu type characterization for the classes $N$ and $B$.

In the sequel, for a function $f$ meromorphic on $D$, we shall denote (see [17])

$$
f_{w}(z)=\frac{f\left(\psi_{w}(z)\right)-f(w)}{1+\overline{f(w)} f\left(\psi_{w}(z)\right)}, \quad \psi_{w}(z)=\frac{z+w}{1+\bar{w} z} .
$$

THEOREM 9. Let $f$ be meromorphic on $D$. Then, the following statements are equivalent.

(i) $f \in N$.

(ii) $\sup _{w \in D} T^{*}\left(f_{w}, 1, p\right)<\infty, p \in(1, \infty)$.

(iii) $\sup _{w \in D} \frac{1}{\pi} \int_{0}^{1} \log ^{p-1}\left(\frac{1}{t}\right) s\left(f_{w}, t\right) \frac{d t}{t}<\infty, p \in(0, \infty)$.

PROOF. Step 1: (i) $\Leftrightarrow$ (ii).

If $f \in N$, then, for $p \in(1, \infty)$,

$$
\begin{aligned}
\sup _{w \in D} \int_{D} f^{\sharp}(z)^{2} \log ^{p}\left|\frac{1-\bar{w} z}{w-z}\right| d m(z) & \leq \sup _{w \in D} \int_{D}\|f\|_{N}^{2} \log ^{p}\left|\frac{1-\bar{w} z}{w-z}\right| \frac{d m(z)}{\left(1-|z|^{2}\right)^{2}} \\
& \leq 2 \pi\|f\|_{N}^{2} \int_{0}^{1} \frac{t}{\left(1-t^{2}\right)^{2}} \log ^{p} \frac{1}{t} d t<\infty .
\end{aligned}
$$

Conversely, if the first expression in the above inequalities is finite, then

$$
\sup _{w \in D} \int_{D} f^{\sharp}(z)^{2} \log ^{p}\left|\frac{1-\bar{w} z}{w-z}\right| d m(z) \geq \sup _{w \in D} \int_{|w-z| /|1-\bar{w} z|<r} f^{\sharp}(z)^{2} \log ^{p} \frac{1}{r} d m(z) .
$$

Hence, there is an $r \in(0,1)$ such that

$$
\sup _{w \in D} \int_{|w-z| /|1-\bar{w} z|<r} f^{\sharp}(z)^{2} d m(z)<\pi .
$$


From this inequality and Yamashita's criterion [26, Lemma 3.2], we have $f \in N$.

Also, since

$$
\left(f \circ \psi_{w}\right)^{\sharp}=f_{w}^{\sharp},
$$

(see, [18, p. 261]), it follows that

$$
\begin{aligned}
\int_{D} f^{\sharp} & (z)^{2} \log ^{p}\left|\frac{1-\bar{w} z}{w-z}\right| d m(z) \\
& =\int_{D} f_{w}^{\sharp}(z)^{2} \log ^{p} \frac{1}{|z|} d m(z) \\
& =\int_{0}^{2 \pi} \int_{0}^{1} f_{w}^{\sharp}\left(r e^{i \theta}\right)^{2} \log ^{p}\left(\frac{1}{r}\right) r d r d \theta \\
& =\int_{0}^{2 \pi} \int_{0}^{1} f_{w}^{\sharp}\left(r e^{i \theta}\right)^{2} p\left[\int_{r}^{1} \log ^{p-1}\left(\frac{1}{t}\right) \frac{d t}{t}\right] r d r d \theta \\
& =p \int_{0}^{2 \pi} \int_{0}^{1}\left[\int_{0}^{t} f_{w}^{\sharp}\left(r e^{i \theta}\right)^{2} r d r\right] \log ^{p-1}\left(\frac{1}{t}\right) \frac{d t}{t} d \theta \\
& =p \int_{0}^{1}\left[\int_{0}^{2 \pi} \int_{0}^{t} f_{w}^{\sharp}\left(r e^{i \theta}\right)^{2} r d r d \theta\right] \log ^{p-1}\left(\frac{1}{t}\right) \frac{d t}{t} \\
& =p \int_{0}^{1}\left[\int_{|z|<t} f_{w}^{\sharp}(z)^{2} d m(z)\right] \log ^{p-1}\left(\frac{1}{t}\right) \frac{d t}{t},
\end{aligned}
$$

for all $p \in(0, \infty)$. Combining the above equations, we arrive at (i) $\Leftrightarrow$ (ii).

Step 2: (i) $\Leftrightarrow$ (iii).

Note that (iii) $\Rightarrow$ (i) is easy. Indeed, suppose, for some fixed $p \in(0, \infty)$,

$$
C=\sup _{w \in D} \frac{1}{\pi} \int_{0}^{1} \log ^{p-1}\left(\frac{1}{t}\right) s\left(f_{w}, t\right) \frac{d t}{t}<\infty .
$$

Then, for each $r \in(0,1)$, and $w \in D$,

$$
C \pi \geq \int_{r}^{1} \log ^{p-1}\left(\frac{1}{t}\right) s\left(f_{w}, t\right) \frac{d t}{t} \geq \frac{s\left(f_{w}, r\right)}{p} \log ^{p} \frac{1}{r} .
$$

Therefore, there is an $r_{0} \in(0,1)$ such that

$$
\sup _{w \in D} s\left(f_{w}, r_{0}\right)<\pi .
$$

By a result of Yamashita [27, Theorem 2], then, $f \in N$.

Conversely, suppose $f \in N$. It is known that (i) $\Leftrightarrow$ (ii). Further, (ii) $\Rightarrow$ (iii) from the inequality $S\left(f_{w}, t\right) \geq s\left(f_{w}, t\right)$, and thus (i) $\Rightarrow$ (iii). This completes the proof. 
THEOREM 10. Let $f \in N$. Then, the following statements are equivalent:

(i) $f \in N_{0}$;

(ii) $\lim _{|w| \rightarrow 1} T^{*}\left(f_{w}, 1, p\right)=0, p \in(1, \infty)$;

(iii) $\lim _{|w| \rightarrow 1} \frac{1}{\pi} \int_{0}^{1} \log ^{p-1}\left(\frac{1}{t}\right) s\left(f_{w}, t\right) \frac{d t}{t}=0, p \in(0, \infty)$.

PROOF. In view of the proof of the previous theorem, we need only verify that (iii) $\Leftrightarrow(\mathrm{i})$.

Suppose, then, that (iii) holds. Then, we can find $r_{1} \in(0,1)$, such that $s\left(f_{w}, r_{1}\right) \rightarrow$ 0 , as $|w| \rightarrow 1$ and hence $f \in N_{0}$, from

$$
f_{w}^{\sharp}(z)=\left(f\left(\frac{w+z}{1+\bar{w} z}\right)\right)^{\sharp}
$$

and Aulaskari's criterion [1, Theorem 2].

The proof that (i) $\Rightarrow$ (iii) is the same as for Theorem 9 .

REMARK 4. For $p \in(0, \infty)$, denote by $Q_{p}^{\sharp}$ or $Q_{p, 0}^{\sharp}$ the set of all meromorphic functions $f$ on $D$ with

$$
\sup _{w \in D} \int_{D} f^{\sharp}(z)^{2} \log ^{p}\left|\frac{1-\bar{w} z}{w-z}\right| d m(z)<\infty,
$$

respectively,

$$
\lim _{|w| \rightarrow 1} \int_{D} f^{\sharp}(z)^{2} \log ^{p}\left|\frac{1-\bar{w} z}{w-z}\right| d m(z)<\infty .
$$

Then, we see from the above argument that, for $p \in(0, \infty)$, we have

$$
f \in Q_{p}^{\sharp} \text { if and only if } \sup _{w \in D} T^{*}\left(f_{w}, 1, p\right)<\infty,
$$

respectively,

$$
f \in Q_{p, 0}^{\sharp} \text { if and only if } \lim _{|w| \rightarrow 1} T^{*}\left(f_{w}, 1, p\right)=0 .
$$

The case $p=1$ is essentially due to Yamashita [26]. In addition, our argument for (i) $\Leftrightarrow$ (ii) in fact gives a new proof for $Q_{p}^{\sharp}=N$ or $Q_{p, 0}^{\sharp}=N_{0}$, whenever $p>1$ (see [2]). In particular, if we denote by $U M$ the class of univalent meromorphic functions in $D$, then, for all $p \in(0, \infty)$,

$$
Q_{p}^{\sharp} \cap U M=U M \text { and } Q_{p, 0}^{\sharp} \cap U M=N_{0} \cap U M .
$$


For a holomorphic function $f$ on $D$, we write

$$
f^{w}(z)=f\left(\psi_{w}(z)\right)-f(w) .
$$

Also, let

$$
T_{*}(f, r, p)=\frac{1}{\pi} \int_{0}^{r} \log ^{p-1}\left(\frac{1}{t}\right) A(f, t) \frac{d t}{t}
$$

where

$$
A(f, t)=\int_{|z|<t}\left|f^{\prime}(z)\right|^{2} d m(z)
$$

which is bigger than

$$
a(f, t)=\int_{f(|z|<t)} d m(z) .
$$

Of course, the last two expressions are the area of $f(|z|<t)$ with (respectively without) multiplicity.

THEOREM 11. Let $f$ be holomorphic on $D$. Then, the following statements are equivalent:

(i) $f \in B$;

(ii) $\sup _{w \in D} T_{*}\left(f^{w}, 1, p\right)<\infty, p \in(1, \infty)$;

(iii) $\sup _{w \in D} \int_{0}^{1} \log ^{p-1}\left(\frac{1}{t}\right) a\left(f^{w}, t\right) \frac{d t}{t}<\infty, p \in(0, \infty)$.

PROOF. The argument is completely similar to that of the previous theorem. However, in the present situation, we make use of a result of Axler [4, Theorem 1], namely,

$$
f \in B \Leftrightarrow \sup _{w \in D} A\left(f^{w}, r\right)<\infty, \text { for each } r \in(0,1) .
$$

In addition, the following inequality is used:

$$
\left|f^{w}(z)\right| \leq \frac{\|f\|_{B}}{2} \log \frac{1+|z|}{1-|z|}, \quad z \in D .
$$

We leave the details to the reader.

THEOREM 12. Let $f \in B$. Then the following statements are equivalent:

(i) $f \in B_{0}$;

(ii) $\lim _{|w| \rightarrow 1} T_{*}\left(f^{w}, 1, p\right)=0, p \in(1, \infty)$; 
(iii) $\lim _{|w| \rightarrow 1} \int_{0}^{1} \log ^{p-1}\left(\frac{1}{t}\right) a\left(f^{w}, t\right) \frac{d t}{t}=0, p \in(0, \infty)$.

PRoof. This follows easily from (5) and the idea in the proof of Theorem 10.

REMARK 5. Let $Q_{p}$ be the set of all holomorphic functions $f$ in $D$, with

$$
\sup _{w \in D} \int_{D}\left|f^{\prime}(z)\right|^{2} \log ^{p}\left|\frac{1-\bar{w} z}{w-z}\right| d m(z)<\infty .
$$

and let

$$
Q_{p, 0}=\left\{f \in Q_{p}: \lim _{|w| \rightarrow 1} \int_{D}\left|f^{\prime}(z)\right|^{2} \log ^{p}\left|\frac{1-\bar{w} z}{w-z}\right| d m(z)=0\right\}
$$

Then,

$$
f \in Q_{p} \Leftrightarrow \sup _{w \in D} T_{*}\left(f^{w}, 1, p\right)<\infty
$$

and

$$
f \in Q_{p, 0} \Leftrightarrow \lim _{|\boldsymbol{w}| \rightarrow 1} T_{*}\left(f^{w}, 1, p\right)=0,
$$

which may be considered as a characterization of Ahlfors-Shimizu type for $Q_{p}$ functions.

The case $p=1$ is due to Yamashita [26, p. 365]. Of course, our idea in proving (i) $\Leftrightarrow$ (ii) can be used to prove again that $Q_{p}=B$ and $Q_{p, 0}=B_{0}$, for $p>1$ [2]. The case $Q_{2}=B$ and $Q_{2,0}=B_{0}$ was first proved in [24] and [25].

In addition, since $f \in Q_{p, 0} \Leftrightarrow f \in Q_{p}$ and

$$
\lim _{|w| \rightarrow 1} \int_{D}\left|f^{\prime}(z)\right|^{2}\left(1-\left|\frac{w-z}{1-\bar{w} z}\right|^{2}\right)^{p} d m(z)=0,
$$

the same idea as in the proof of (i) $\Leftrightarrow$ (ii) of Theorem 12 yields that $f \in Q_{p, 0}$ if and only if

$$
\lim _{|w| \rightarrow 1} \int_{0}^{1}(1-t)^{p-1} A\left(f^{w}, t\right) d t=0, \quad p \in(0, \infty)
$$

and $f \in B_{0}$ if and only if

$$
\lim _{|w| \rightarrow 1} \int_{0}^{1}(1-t)^{p-1} a\left(f^{w}, t\right) d t=0, \quad p \in(0, \infty) .
$$

This gives a complete answer to the question which remained open in [23]. 
REMARK 6. Let $U H$ denote the class of univalent holomorphic functions on $D$. Then,

$$
Q_{p} \cap U H=B \cap U H \text { and } Q_{p, 0} \cap U H=B_{0} \cap U H .
$$

See $[3$, Theorem 6.1].

\section{References}

[1] R. Aulaskari, 'On the boundary behaviour of Bloch and normal functions', Bull. Austral. Math. Soc. 30 (1984), 299-305.

[2] R. Aulaskari and P. Lappan, 'Criteria for an analytic function to be Bloch and a harmonic or meromorphic function to be normal', in: Complex analysis and its applications, Pitman Res. Notes Math. 305 (Longman Sci. Tech., Harlow, 1994) pp. 136-146.

[3] R. Aulaskari, P. Lappan, J. Xiao and R. Zhao, 'On $\alpha$-Bloch spaces and multipliers of Dirichlet spaces', J. Math. Anal. Appl. 209 (1997), 103-121.

[4] S. Axler, 'The Bergman space, the Bloch space, and commutators of multiplication operators', Duke Math. J. 53 (1986), 315-332.

[5] S. Axler and K. Zhu, 'Boundary behavior of derivatives of analytic functions', Michigan Math. $J$. 39 (1992), 129-143.

[6] A. Baernstein II, 'Analytic functions of bounded mean oscillation', in: Aspects of contemporary complex analysis (eds. D. A. Brannan and J. G. Clunie) (Academic Press, London, 1980) pp. 3-36.

[7] H. Brezis and L. Nirenberg, 'Degree theory and $B M O$; Part II: Compact manifolds with boundaries', Selecta Math., Soviet New Ser. 2 (1996), 309-368.

[8] L. Brown and P. M. Gauthier, 'Behavior of normal meromorphic functions on the maximal ideal space of $H^{\infty}$, Michigan Math. J. 18 (1971), 365-371.

[9] L. Carleson, 'Interpolation by bounded analytic functions and the corona problem', Ann. of Math. 76 (1962), 547-559.

[10] R. Coifman, R. Rochberg and G. Weiss, 'Factorization theorems for Hardy spaces in several complex variables', Ann. of Math. 103 (1976), 611-635.

[11] J. Garnett, Bounded analytic functions (Academic Press, New York, 1981).

[12] K. Hoffman, 'Bounded analytic functions and Gleason parts', Ann. of Math. 86 (1969), 74-111.

[13] P. Lappan, 'Non-normal sums and products of unbounded normal functions', Michigan Math. J. 8 (1961), 187-192.

[14] — 'Some results on harmonic normal functions', Math. Z. 90 (1965), 155-159.

[15] O. Lehto and K. I. Virtanen, 'Boundary behaviour and normal meromorphic functions', Acta Math. 97 (1951), 47-65.

[16] K. Noshiro, 'On the theory of cluster sets of analytic functions', J. Fac. Sci. Hokkaido Univ. 7 (1938), 149-159.

[17] Ch. Pommerenke, 'Estimates for normal functions', Ann. Acad. Sci. Fenn. Ser A I Math. 476 (1970), 1-10.

[18] — Univalent functions (Vandenhoeck and Ruprecht, Göttingen, 1975).

[19] H. M. Reimann, 'Functions of bounded mean oscillation and quasiconformal mappings', Comm. Math. Helv. 49 (1974), 260-276.

[20] H. M. Reimann and T. Rychener, Funktionen beschrankter mittlerer Ozillation, Lect. Notes in Math. 487 (Springer, Heidelberg, 1975). 
[21] J. L. Schiff, Normal families (Springer, Heidelberg, 1993).

[22] K. Stroethoff, 'Nevanlinna-type characterizations for the Bloch space and related spaces', Proc. Edinburgh Math. Soc. 33 (1990), 123-141.

[23] K. Wirths and J. Xiao, 'Image areas of functions in the Dirichlet type spaces and their Möbius invariant subspaces', Maria Curie-Slowdkowska, Sect. A 22 (1996), 239-245.

[24] J. Xiao, 'Carleson measure, atomic decomposition and free interpolation from Bloch space', Ann. Acad. Sci. Fenn. Ser. A I Math. 19 (1994), 35-46.

[25] J. Xiao and L. Zhong, 'On little Bloch space and its Carleson measure, atomic decomposition and free interpolation', Complex Variables 27 (1995), 175-184.

[26] S. Yamashita, 'Functions of uniformly bounded characteristic', Ann. Acad. Sci. Fenn. Ser. A IMath. 7 (1982), 349--367.

[27] - 'Area criteria for functions to be Bloch, normal and Yosida', Proc. Japan Academy 59 (1983), 462-464.

[28] K. Zhu, Operator theory in function spaces, Pure and Applied Math. 139 (Marcel Dekker, New York, 1990).

Département de mathématiques et de statistique School of Mathematical Sciences Université de Montréal Peking University

Montréal H3C3J7 Beijing 100871

Canada

P. R. China

e-mail: gauthier@dms.umontreal.ca

e-mail: jxiao@sxxxo.math.pku.edu.cn 